Questions de communication

$24 \mid 2013$

Renouvellement des mises en scène télévisuelles de la politique

Otto B. KRAUS, Le Mur de Lisa Pomnenka, suivi de Catherine COQUIO, Le Leurre et l'espoir. De Theresienstadt au block des enfants de Birkenau Paris, Éd. L'Arachnéen, 2013, 366 pages

Carine Trevisan

\title{
OpenEdition
}

Journals

Édition électronique

URL : http://journals.openedition.org/questionsdecommunication/8801

DOI : 10.4000/questionsdecommunication.8801

ISSN : 2259-8901

Éditeur

Presses universitaires de Lorraine

Édition imprimée

Date de publication : 31 décembre 2013

Pagination : 285-287

ISBN : 978-2-8143-0182-5

ISSN : $1633-5961$

Référence électronique

Carine Trevisan, «Otto B. kraus, Le Mur de Lisa Pomnenka, suivi de Catherine coauıo, Le Leurre et l'espoir. De Theresienstadt au block des enfants de Birkenau », Questions de communication [En ligne], 24 | 2013, mis en ligne le 01 février 2014, consulté le 22 septembre 2020. URL : http://journals.openedition.org/ questionsdecommunication/8801; DOI : https://doi.org/10.4000/questionsdecommunication.8801 
ils choisissent de démontrer « que le sport, né avec le capitalisme, n'a pas connu d'âge d'or mythique, qu'il n'a jamais été l'outil de développement de quelque humanisme, qu'il n'a jamais été une ode ou une poésie ailleurs que dans le fantasme » (p. 10). Cette vision critique et déshumanisée du sport le rend totalement étranger à toute forme de beauté, de transcendance. Si le positionnement initial des auteurs est intéressant, leur jusqu'au-boutisme les enferme dans une sociologie étroite et marxisante, dans une vision étriquée de la réalité du sport qui transparaît - et de quelle manière - dans le développement suivant: « Nous croyons [...] que le sport s'attaque à ce que nous pourrions nommer la société, une société que nous imaginons globale dans le sens où celle-ci serait utopiquement démocratique, c'est-à-dire un système réalisé par le peuple, pour le peuple, avec le peuple, dans un environnement respecté, renouvelable, donc renouvelé » (ibid.). Si l'angle d'attaque semble intéressant, l'outrance qui le sous-tend est parfois dérangeante. Ainsi le sport serait-il une atteinte à la démocratie, un système autocratique et antisocial ? Voici comment les auteurs défendent et clarifient leur problématisation scientifique : « Le sport est contre la société. II lutte contre toutes les utopies démocratiques et participe de l'altération et de l'accroissement antidémocratique en proposant une philosophie de la sélection, donc de l'exclusion, dans une lutte de tous contre tous, de chacun contre tous et de tous contre chacun » (ibid.). Pour radicale qu'elle soit, cette thèse mérite de dépasser le stade de l'autoaffirmation et d'être examinée scientifiquement.

Dans le premier chapitre (pp. 13-40) - le plus intéressant de l'ouvrage et de loin -, Patrick Vassort établit un parallèle entre le sport et la domination capitaliste, rappelant justement que l'Europe est à la fois « le continent le plus riche et le plus anciennement capitaliste, le continent de l'invention du sport, le continent de l'invention de la compétition totale, globale » (p. 13). Non seulement, l'auteur ne perçoit pas le sport comme une alternative à la crise, mais il le considère comme une pratique portant en germe toutes les crises économiques et politiques actuelles. II cite le discours de l'ancien président de la République française, Nicolas Sarkozy, en 2010, à la tribune de l'Union européenne des associations de football qui a permis d'obtenir que la France organise le Championnat d'Europe des nations 2016: « Nous pensons en France que le sport est une réponse à la crise. C'est justement parce qu'll y a une crise, qu'il y a des problèmes, qu'il faut mobiliser tout un pays vers l'organisation des grands évènements [...]. C'est une décision stratégique qui engage tout le pays face à la crise [...]. Ce n'est pas un engagement de la Fédération, ce n'est pas un engagement de la Ligue, c'est un engagement de tout un peuple » (Le Point, 28/05/10, pp. 14-15). Ce discours est symptomatique de l'importance que le sport revêt dans l'imaginaire politique - y compris au plus haut sommet de l'État et de la tentation d'en détourner les valeurs au profit de la lutte politicienne. Néanmoins, Patrick Vassort rappelle qu'il aura fallu « trois décennies à Montréal (1976-2006) pour rembourser les dettes engendrées par l'organisation de Jeux olympiques d'été ») (p. 18) et que l'organisation de ceux de 2004 aura précipité la Grèce dans une dépression économique d'une violence inouie. L'auteur ajoute que, très souvent, l'organisation d'une compétition sportive internationale fait presque naturellement taire les oppositions aux pouvoirs politiques les moins acceptables et que, en alléguant de fausses raisons (sécurité, propreté, rénovation), ces pouvoirs arbitraires n'hésitent pas à détruire des quartiers entiers et à déplacer des milliers de personnes contre leur gré pour réaliser de gros profits (il n'est qu'à voir le récent exemple de la ville russe de Sotchi qui accueillera les Jeux olympiques d'hiver en 2014 et qui a été complètement défigurée par les travaux).

Dans la conclusion de l'ouvrage, les trois directeurs rappellent que le sport a colonisé la vie dans sa totalité et surtout ses espaces les plus politiques: l'espace public et les lieux des nouvelles urbanisations, l'espace de ce qui devrait être les lieux d'expression de la pensée politique (télévision, magazines, radios, journaux, etc.), les parlements, jusque dans ses espaces les plus intimes - ceux de l'éducation et de la transcendance culturelle -, ceux de la sexualité et des pulsions, ceux du corps. Face à cette inquiétante déferlante, à cette véritable pandémie, - à en croire ces auteurs - rien ne peut apparemment subsister. Ce constat, alarmant et alarmiste, n'enlève cependant rien à la finesse et à la profondeur de certaines analyses qui émaillent un ouvrage par trop inégal.

Alexandre Eyries

13M, université Nice Sophia Antipolis, F-06200 alex.eyries@yahoo.fr

\section{Otto B. Kraus, Le Mur de Lisa Pomnenka, suivi de Catherine CoQuio, Le Leurre et P'espoir. De Theresienstadt au block des enfants de Birkenau.}

Paris, Éd. L'Arachnéen, 2013, 366 p.

« La guerre que fit Adolf Hitler à la plupart des pays européens pour imposer "l'ordre nazl" [... fut également la première guerre de l'histoire consciemment livrée aux enfants » (Félix Tych, Archives clandestines du ghetto de Varsovie, Paris, Fayard/BDIc, 2007, 
cité in : Catherine Coquio, Aurélia Kalisky, L'Enfant et le génocide, témoignages sur l'enfance pendant la Shoah, Paris, R. Laffont, 2007, p. 15). Co-auteure avec Aurélia Kalisky d'une monumentale anthologie publiée sous le titre L'Enfant et le génocide, témoignages sur l'enfance pendant la Shoah, Catherine Coquio commente ici, dans la continuité de lintérêt qu'elle porte au sort faits aux enfants sous l'Allemagne nazie, un texte d'Otto B. Kraus : Le mur de Lisa Pomnenka. Le livre se situe entre le témoignage et la fiction. II raconte la façon dont des enfants, destinés à la mort dans le camp de Birkenau, ont vécu quelques mois « à l'ombre » des crématoires presque comme si de rien n'était. Ces enfants ont développé une étonnante capacité à jouer, à rêver - « une autre liberté dont les SS ne pouvaient les priver était celle de rêver » (p. I 17) -, une curiosité et un désir d'apprendre - « Ici le prisonniern I 70 563, qui joue aux billes ou saute à la corde ou apprend les noms des jours de la semaine en allemand » (pp. I I2) -,élans entretenus par des " éducateurs 》, eux-mêmes voués à être exterminés. Dans le « block des enfants » de Birkenau, les jeux, les spectacles de théâtre, les activités poétiques étaient autorisés, mais on y interdisait toute forme d'enseignement. Les enfants qui n'étaient pas orphelins pouvaient rejoindre, pendant la nuit, leurs mères dans un bloc voisin. Comme le note Catherine Coquio dans l'essai remarquablement documenté qui accompagne le texte, ce bloc des enfants avait l'aspect d'une sorte dîle ou de bateau en naufrage dont les passagers feignent d'ignorer ce qui les attend. Une feinte « entre le leurre et l'espoir », pour reprendre son expression.

Le leurre apparât très vite comme celui qui est imposé par les nazis. Le bloc des enfants de Birkenau a été conçu, dans l'attente d'une visite de la CroixRouge, comme le prolongement du ghetto-vitrine de Theresienstadt, en Tchécoslovaquie. L'histoire est connue, on en a fait un film de propagande, tourné en 1944, sous le titre Le Führer offre une ville aux Juifs (Kurt Gerron). Le ghetto de Terezin - où étaient entassées 50000 personnes - fut embelli fin 1943, les déportés les moins « présentables » furent soustraits aux regards. On fit ouvrir un jardin d'enfants, jouer un opéra d'enfants, genre qu'appréciaient particulièrement les nazis, comme le note Catherine Coquio. Des extraits de ce film ont été magnifiquement commentés par l'écrivain Winfried G. Sebald dans Austerlitz (trad. de l'allemand par Patrick Charbonneau, Paris, Gallimard, 2006 [200 l]), où il cherche à retrouver le visage de sa mère, déportée dans ce ghetto.

À Birkenau, il semble que l'espoir prévale sur le leurre. Lisa Pomnenka, qui donne son titre au livre, est une jeune femme juive qui, en échange de portraits de tsiganes et d'arbres généalogiques que lui commande Josef Mengele (« médecin » du camp, qui pratique, on le sait, la vivisection sur les corps humains) obtient les matériaux nécessaires pour peindre un mur du bloc des enfants. Un mur où elle tente de faire exister ce que les enfants n'ont jamais vu : des arbres, des chats, des vaches, des poules, des oiseaux, des fleurs. « Je peindrai, dit Lisa, tout ce qui nous manque ici » (p. 76). Auprès de ce mur, est créée une « bibliothèque ambulante ». Les adultes s'occupant des enfants tentent de se remémorer les livres qu'ils ont lus, qu'ils leur racontent - on songe ici à Farenheit 45I (Ray Bradbury, trad. de l'américain par Henri Robillot, Paris, Denoël, 1955 [1953]) où la question se pose de savoir que faire quand il n'y a plus de livres, chacun explorant sa mémoire des livres lus. Un extraordinaire art de la pénurie s'invente, développé par les adultes, encouragé par les enfants.

Les pages sans doute les plus bouleversantes de l'ouvrage sont celles du récit de la célébration de Pessah, fête juive commémorant la sortie d'Egypte, le passage, pour les Juifs, de l'esclavage à la liberté. Pour cette célébration, les gâteaux sont faits de confiture de betteraves. Le thé fait office de vin. Les enfants détenus chantent l'Ode à la joie de Beethoven : « À ce moment précis, ils avaient vaincu les Allemands 》 (p. 95). Lîle de Birkenau devient «lî̀e de Robinson »: la preuve d'une extraordinaire capacité de résistance. La vitalité étonnante des enfants semble alimenter celle des adultes chargés de s'occuper d'eux: ils préparent une révolte, ils gardent la capacité d'être amoureux et désirants.

Reste que les enfants ne sont pas dupes. Ils savent quills vont mourir et que tout le monde fait « comme si... 》, sur le modèle, peut-être, d'Alice au Pays des merveilles: «let's pretend... » Certains enfants font « comme si... 》, mais d'autres renoncentà à'illusion :ainsi le petit Adam qui, pour survivre, devient Piepel (objet sexuel du kapo) tout en écrivant des poèmes fait-il songer à ceux qu'évoque Jean Cayrol dans « les rêves concentrationnaires 》 (Les Temps modernes, 36, sept. 1946, pp. 520-535), des rêves de couleur: « Un monde vert/Avec une porte verte/et un oiseau au plumage vert » (p. 82). À la fin d'un conte triste, la petite fille note-t-elle: « Ils n'étaient pas pauvres. Ils pouvaient marchander leur pain et leurs lentilles » (p. 109). La première prière juive que ces enfants entendent est celle du kaddish, la prière pour le mort...

Dans l'essai qui accompagne ce texte, Le leurre et l'espoir. De Theresienstadt au block des enfants de Birkenau, Catherine Coquio fait une histoire très précise de ce livre paru à Prague en 1993 sous le titre Mon frère en fumée, puis en Israël, en 1995, sous 
le titre The Painted Wall. L'auteur, jeune homme juif tchèque a vécu de décembre 1943 à juillet 1944 dans le bloc des enfants de Birkenau, où il était chargé de les encadrer. Le camp, et en particulier ce bloc, était contrôlé par Josef Mengele avec l'approbation d'Adolf Eichmann. Les enfants ont été « liquidés 》 six mois après leur arrivée, en juillet 1944. Otto B. Kraus écrit un journal fictif, sous le couvert d'Alex Ehren, exécuté lors d'une marche de la mort.

Catherine Coquio, et c'est là l'un des aspects les plus intéressants de l'essai, montre combien le texte d'Otto B. Kraus est important parce qu'il touche non seulement le sort des enfants au Xxe siècle, mais « la mémoire plombée du génocide » (p. 232) dans les « démocraties populaires » (ibid.) sous l'égide $d u$ communisme soviétique. En 1959, sur l'un des murs d'une synagogue de Prague furent gravés les noms de 77297 juifs tchèques assassinés. En 1968, après l'invasion soviétique, ces noms furent effacés...

Carine Trevisan

CERILAC, université Paris Diderot-Paris 7 carine.trevisan@univ-paris-diderot.fr

\section{Pascal Pansu, Nicole Dubols, Jean-Léon Beauvols, Dis- moi qui te cite et je saurai ce que tu vaux. Que mesure vraiment la bibliométrie?}

Grenoble, Presses universitaires de Grenoble, coll. Points de vue et débats scientifiques, 2013, I27 p.

À la lecture de l'ouvrage, on ne peut s'empêcher de penser à la difficulté évoquée par Pierre Bourdieu dans Homo academicus (Paris, Éd. de Minuit, 1984, p. I I) de conduire une réflexion critique et distanciée au sujet $d^{\prime}$ « un monde social dans lequel on est pris ». C'est pourtant l'exercice auquel se prêtent Pascal Pansu, Nicole Dubois et Jean-Léon Beauvois dans le livre, même si celui-ci constitue plus un état des lieux des principes qui gouvernent la scientométrie, qu'une diatribe sociologique. Pourtant, on y trouvera non seulement de quoi nourrir une réflexion questionnant le sens d'une course effrénée à la publication qui régit de longue date le monde académique, mais également quelques réponses concrètes. Dès l'introduction (pp. 5-8), les auteurs annoncent la position qui sera la leur : une position nuancée qui ne conteste pas la nécessité de l'évaluation de la recherche universitaire, mais qui entend poser un regard éclairé et critique sur les modalités de cette évaluation. D'emblée, la posture de détracteurs farouches du facteur d'impact et d'autres indicateurs bibliométriques est donc rejetée.
Dans le premier chapitre, «L'évaluation comme nécessité organisationnelle » (pp. 9-16), l'évaluation est présentée comme une « nécessité sociale » dans la mesure où les chercheurs, en tant qu'« agents sociaux en relation d'équivalence » (p. I I), forment un pouvoir social qui doit se laisser évaluer. Si l'évaluation répond à des besoins organisationnels, mais aussi à des besoins institutionnels de compétitivité, elle nécessite que chacun « entre dans le jeu », selon la formule de Pierre Bourdieu (op. cit, p. II7) et en accepte les règles. Dans cette perspective, elle se doit d'être efficace et objective. À ce titre, les auteurs énoncent les questions qui fondent la trame de l'ouvrage : qui doit évaluer la recherche? À quel rythme? Sur quels critères? Enfin, que doit-on évaluer? En réponse à ces différentes questions, les auteurs proposent un état des lieux des outils et des pratiques liées à la bibliométrie d'impact.

De manière synthétique mais complète, le deuxième chapitre, "Une introduction à la bibliométrie d'impact » (pp. 17-44), présente les fondements et le fonctionnement de la scientométrie, discipline qui trouve son origine à l'orée du $x x^{e}$ siècle et connaitt un essor important à partir années 50 aux États-Unis. Fondée sur des méthodes statistiques et mathématiques, la scientométrie permet de mesurer la productivité des chercheurs. Elle se décline en deux volets : l'un descriptif et l'autre évaluatif. La scientométrie descriptive rend compte de la production d'un chercheur dans un domaine donné au cours d'une période déterminée, en fonction de ce que les autres chercheurs ont publié dans ce même domaine au cours de cette même période. Cette approche descriptive n'implique donc pas de jugement de valeur. En outre, elle permet de faire émerger les réseaux disciplinaires par repérage des co-citations et de montrer « la façon dont un champ scientifique se structure et évolue » (p. 43). Quant à la bibliométrie d'impact, elle constitue le volet évaluatif de la scientométrie. En effet, on ne peut évaluer la productivité des chercheurs à l'aune du seul nombre d'articles publiés. II importe également de croiser cette information avec d'autres indicateurs tel le prestige de la revue par exemple. La bibliométrie d'impact étudie l'influence scientifique des chercheurs et l'impact sur leurs pairs en mesurant, par exemple, pour une période donnée, le nombre de citations des articles publiés dans une revue par rapport au nombre total d'articles publiés dans cette même revue - le fameux facteur d'impact. Développées et commercialisées par l'Institute for Scientific Information (III), puis parThomson Reuters, les bases de données bibliographiques Current Contents, Science Citation Index,Social Citation Index, ou encore le Journal Citation Reports constituent autant de produits 